(C)2007 IEEE. Personal use of this material is permitted. However, permission to reprint/republish this material for advertising or promotional purposes or for creating new collective works for resale or redistribution to servers or lists, or to reuse any copyrighted component of this work in other works must be obtained from the IEEE 


\title{
Digital Ecosystem Ontology
}

\author{
Hai Dong, Farookh Khadeer Hussain \\ Digital Ecosystem and Business Intelligence Institute \\ Curtin University of Technology \\ Perth, Australia \\ Email: hai.dong@cbs.curtin.edu.au, farookh.hussain@cbs.curtin.edu.au
}

\begin{abstract}
Digital Ecosystems is a neoteric terminology and there are two major definitions about it respectively from Soluta.Net and from Digital Ecosystem and Business Intelligence Institute. In this paper, to solve the ambiguous problem in Digital Ecosystem's definitions and to help researchers better understand what it is, by means of ontology, we propose a conceptual model to completely illustrate the concepts in Digital Ecosystem. By introducing a new ontology notation system, we deliver the Digital Ecosystem Ontology, to well define the components and explain the relationships between these components. Finally we realize the ontology in Protégé-owl and conclude our future works in the field.
\end{abstract}

\section{INTRODUCTION}

Digital Ecosystems is a neoteric terminology and its appearance is as a result of the natural existence of Business Ecosystem, along with the evolution of business network and information technology. The goal of Digital Ecosystems is to improve the efficiency of the communication between internal agents and to structuralize the existing Business Ecosystem. Because the research on Digital Ecosystem is still in the beginning stage, some basic concepts are in debate. The definition of Digital Ecosystem is one of them, which have two major opinions. One is defined by Solutanet from the structural and functional perspective, which sees Digital Ecosystem as an open-source network environment for business mainly including small and medium enterprises to interact with others in an effective and efficient way [3]. Another one is defined by Digital Ecosystem and Business Intelligence Institute (DEBII) from the perspective of specialization, which sees it as "an open, loosely coupled, domain clustered, demand-driven, self-organizing and agentbased environment which each species is proactive and responsive for its own benefit and profit" [2] [4]. The former definition and related theories focus on the introduction and implementation of the Digital Ecosystem architecture from pure technical aspect. By constructing the service-oriented environment by means of network technology, BML (Business Modeling Languages), SDL (Service Description Languages), ontology et al., Digital Ecosystem realizes the function of service creation, service execution and service optimization [3]. The latter definition and based theories concentrate on defining the existing concepts and network architecture and swarm intelligence in Digital Ecosystem, which tends to conceptualize and structuralize its components. Thus, the former definition is preferable to the actual implementation and commercialization, and the latter definition more inclines to the conceptualization.

To solve the conceptual ambiguity and help researcher better understand what Digital Ecosystem is, we employ ontology which is seen as an affective tool to assist people share knowledge, to represent the conceptual model of Digital Ecosystem. Due to the mission of this paper is to conceptualize the components of Digital Ecosystem, the theory that our research bases mainly concentrates on the second definition we discussed above.

\section{NOTATION SYSTEM FOR ONTOLOGY REPRESENTATION}

Before we represent the ontology model for Digital Ecosystem, a notation system is introduced in this section. The notation system utilized in the ontological representation is based on Chang et al.'s work, which consists of four basic notations as Table 1[1]. Although in the past we usually used to employ UML (Unified Modelling Language) to represent ontology model, due to its complex symbols categories, UML cannot efficiently help people better understand the shared knowledge. This notation system simplifies the symbols and its symbols are closer to the principle of ontology which is the combination of shared concepts and relationships between concepts [5].

TABLE I

ONTOLOGY NOTATION SYSTEM

\begin{tabular}{|l|l|}
\hline Ontology Notation & \multicolumn{1}{|c|}{ Semantics of the Notation } \\
\hline & $\begin{array}{l}\text { Double-field Box represents the Ontological } \\
\text { Concepts. }\end{array}$ \\
\hline & $\begin{array}{l}\text { A dotted line represents Ontology Concept } \\
\text { Association Relation which represents a } \\
\text { Concept is closely related to another concept. } \\
\text { The relationship name can be noted above the } \\
\text { dotted line. }\end{array}$ \\
\hline$\longrightarrow$ & $\begin{array}{l}\text { Open-arrow line represents Composition and } \\
\text { Aggregation or Part-of relationship between } \\
\text { Upper Ontology Concept and Lower Ontology } \\
\text { Concept. }\end{array}$ \\
\hline$\longrightarrow$ & $\begin{array}{l}\text { Solid-arrow line represents Generalization and } \\
\text { Specification relation, which is a relation } \\
\text { between Upper Generic Concept and Lower } \\
\text { Specific Concept. }\end{array}$ \\
\hline
\end{tabular}

III. Digital ECOSYSTEM ONTOLOGY 


\section{A. Hierarchy of Digital Ecosystem Ontology Concepts}

Digital Ecosystem consists of two basic parts - species and an ecosystem environment. Each species can be viewed as an individual or an organisation and has its own role to play. The species works together to take care of their living environment. One specialisation of species is that they are collaborative and usually work in a group, and a leader leads all other followers in the group. According to the definition of digital ecosystem, the environment has the specification of "open, loosely coupled, demand-driven, domain clustered, self-organising and agent-based" [2] [4]. Thus, the hierarchy of Digital Ecosystem concepts is shown in Fig.1 through the use of notation system.

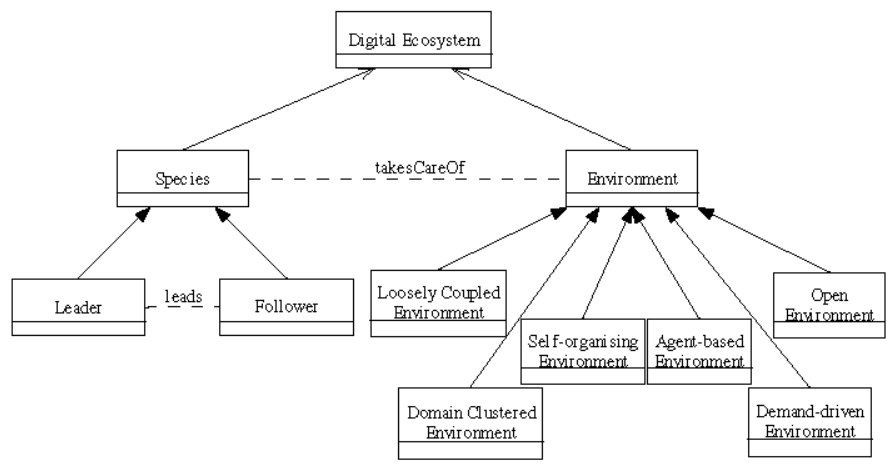

Fig. 1. Digital Ecosystem concepts hierarchy

\section{B. Species Ontology}

Species is the basic element of Digital Ecosystem, which can be defined as the conceptualization of Species which comes from certain Domain, plays dual Roles including the Supplier who has Available Services and the Requester who has Requested Service, follows Rules in Digital Ecosystem, is driven by own Profit and carried out Tasks that relates to the Profit. (Fig. 2)

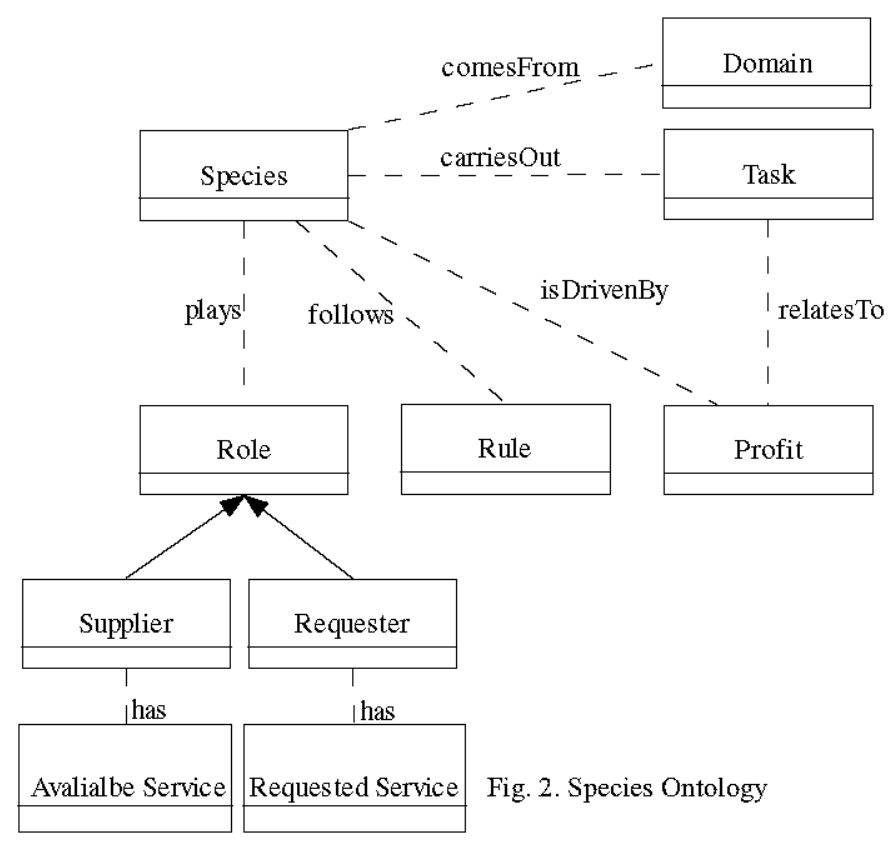

We present the Species Ontology as the combination of ontology name and a tuple where the elements of the tuple can be complex elements as defined below:

Species [Species, Domain, Task, Profit, Rule, Role, Supplier, Requester, Available Service and Requested Service] where

'Species' refers to the definitions above.

'Domain' refers to a field in Digital Ecosystem where a species has common interest with other species.

'Task' refers to a mission that a species is executing, to survive and achieve its goals in the environment of Digital Ecosystem.

'Profit' or 'Benefit' means a social or economical advantage that a species gains by Digital Ecosystem.

'Rule' refers to a common or special regulation that a species should follow in the environment of Digital Ecosystem.

'Role' refers to the role of a species in the interaction with other species. There are two major roles in Digital Ecosystem interactions - Supplier and Requester. Differed from other network architectures in which each agent has fixed role, each species in Digital Ecosystem are able to play the dual roles at the same time.

'Supplier' is a role of a species that provides a service.

'Requester' is a role of a service that requests a service.

'Available Service' is a service that a supplier provides.

'Requested Service' is a service that a requester needs.

\section{Leader Ontology and Follower Ontology}

Prior to defining the Leader Concept and Follower Concept, the organizational structure of species' groups is discussed. In Digital Ecosystem, due to the specification of self-organizing and collaboration, species may form a hierarchical organization of flexible structures. Here a concept - swarm is defined by Chang and West, which is 'a set of species which has common characteristic and is able to interact and engage directly or indirectly with each other' [4]. With swarm intelligence technology, the species that have common interest may group and cooperate, to share a problem and to collaboratively carry out a task.

In a swarm, a leader is selected, to direct the other species' activities and represent the belonged swarm to interact with other swarms. The other species in this warm are directed by the leader and realize their own functions [4].

The subclasses of Species Concept - Leader Concept and Follower Concept inherit all properties from Species Concept. The only difference is the relationship between Leader and Follower, which a leader leads all follower and all followers follow a leader in a swarm.

The ontological representation of Leader Ontology and Follower Ontology is shown in Fig. 3 and Fig.4. 


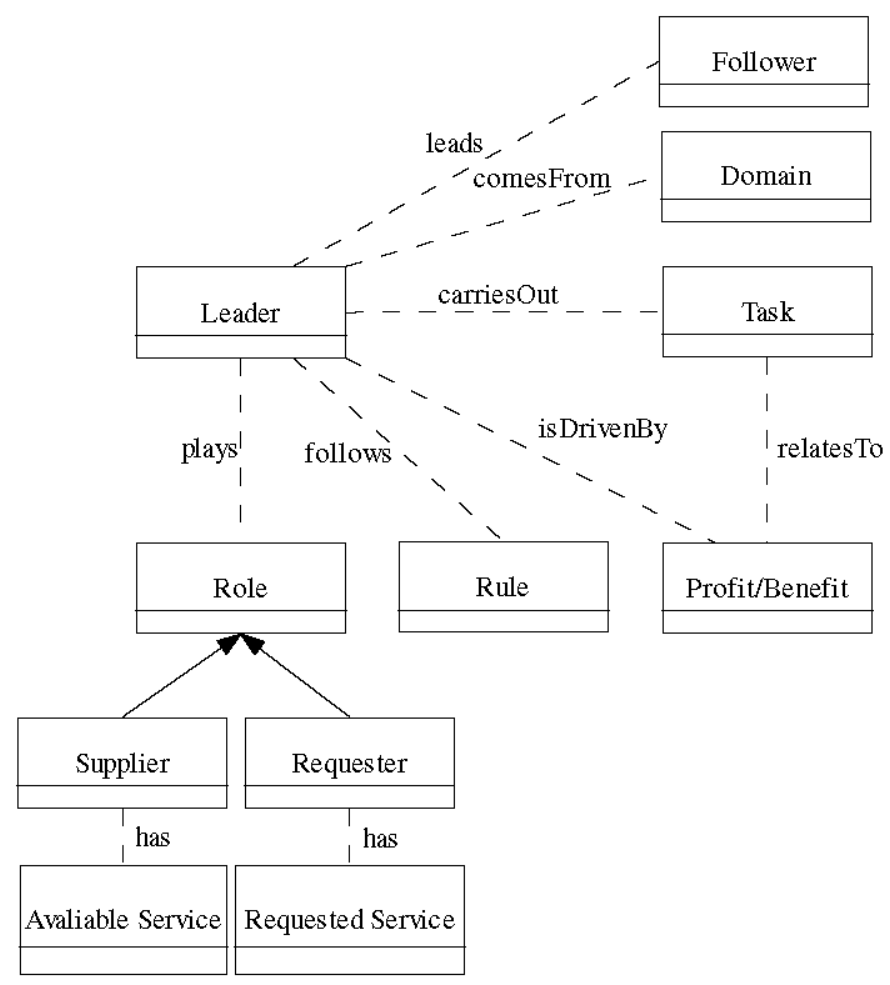

Fig. 3. Leader Ontology

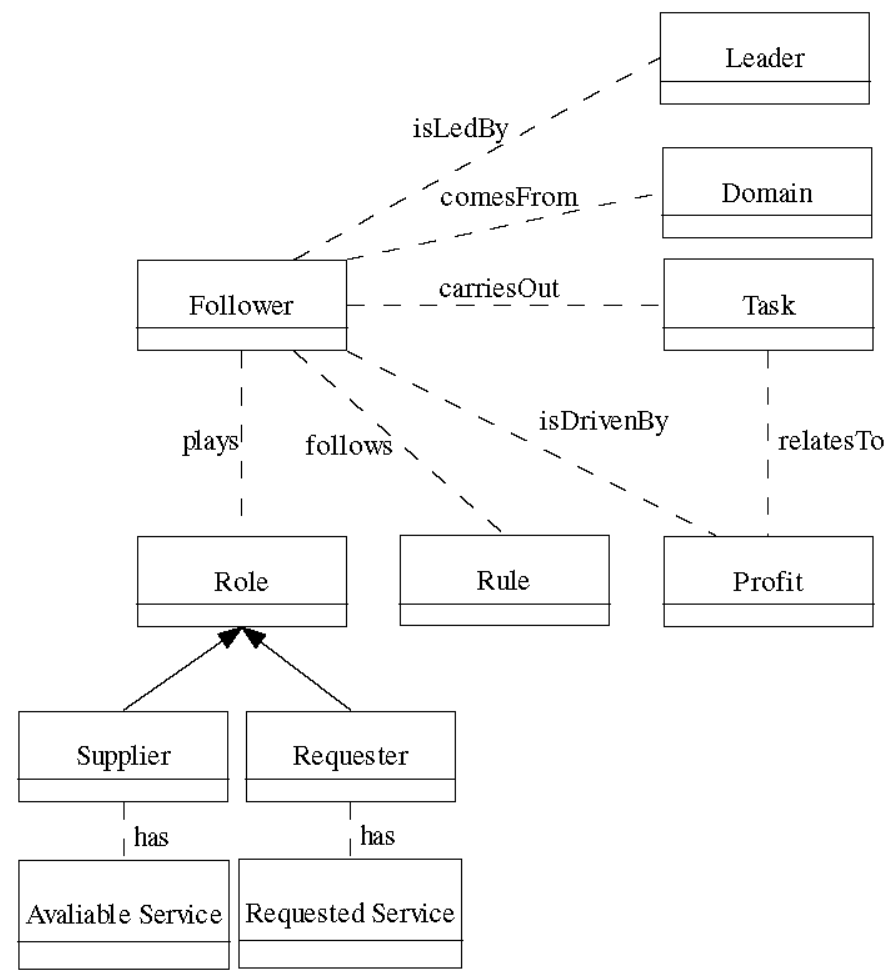

Fig. 4. Follower Ontology

\section{Environment Ontology}

In an ecosystem, the environment supports all basic requirements for species' survival and evolution. However, species do not need to take care of their living environment.
Differed from an ecosystem, there is bidirectional relationship between environment and species in Digital Ecosystem. Apart from the environment as the foundation for species' living which supports services to species, species also need to take care of the environment by means of providing technologies [1].

In the environment of Digital Ecosystem, the environment can be defined as the conceptualization of Environment that is supported by Technologies provided by Species and supports Services to Species and has six subclasses - Open Environment, Loosely Coupled Environment, Demand-driven Environment, Domain Clustered Environment, Self-organizing Environment and Agent-based Environment. The Environment Ontology is shown in Fig. 5 through the use of notation system.

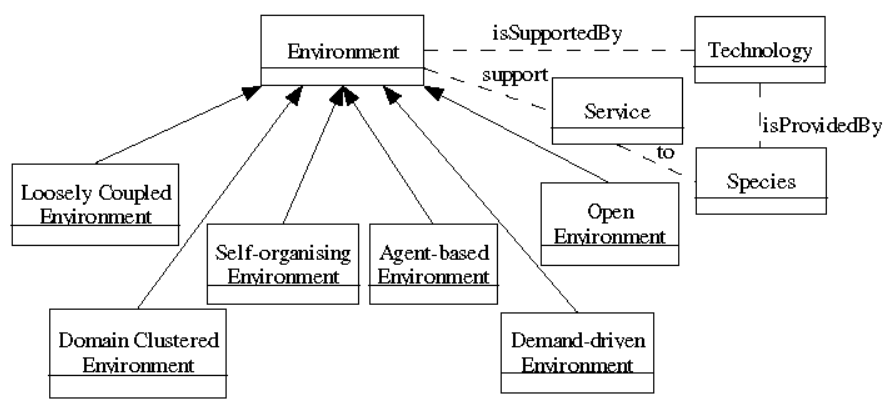

Fig. 5. Environment Ontology

We present the Environment Ontology as the combination of ontology name and a tuple where the elements of the tuple can be complex elements as defined below:

Environment [Environment, Technology, Service, Species, Open Environment, Loosely Coupled Environment, Demanddriven Environment, Domain Clustered Environment, Selforganizing Environment and Agent-based Environment] where

'Environment' refers to definition above.

'Technology' refers to flexible Digital Ecosystem architecture, self-organizing agents, ontology-based knowledge sharing and swarm intelligence in Digital Ecosystem [4].

'Service' refers to the services required by species, including digital services, customer services and agent services [4].

'Species' refers to the definition before.

'Open Environment' refers to a transparent environment where all interactions are visible [4].

'Loosely Coupled Environment' refers to a freely bound and open relationship between species in Digital Ecosystem [4].

'Demand-driven Environment' means that species actively join in a community on their own interest [4].

'Domain Clustered Environment' refers to an environment which consists of the field where some species have common interest [4].

'Self-organizing Environment' means that species are capable of acting autonomously, making decisions and carrying out tasks in Digital Ecosystem [4]. 
'Agent-based Environment' refers to an environment which contains human individuals, information technologies and tools that facilitate interaction and knowledge sharing along with the resources that help maintain synergy among human beings and organizations [4].

\section{PROTOTYPE}

To realize and implement our design, we build the conceptual model by means of Protégé-owl. The graphical view of Hierarchy of Digital Ecosystem Ontology concepts in Protégé-owl is shown in Fig. 6.

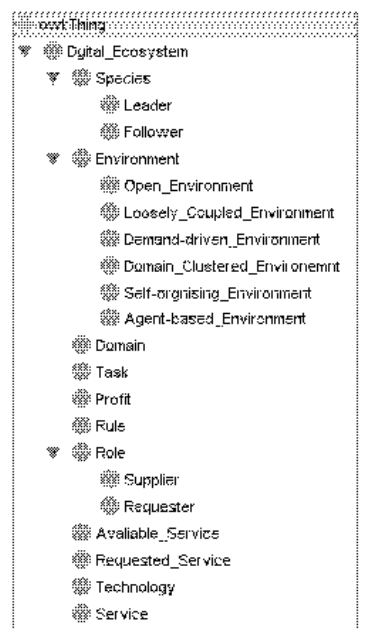

Fig. 6. Hierarchy of Digital Ecosystem Ontology concepts in Protégé-owl

\section{CONCLUSION AND FUTURE WORKS}

In this paper, to solve the ambiguous problem in defining Digital Ecosystem, we use ontology to represent the conceptual model of Digital Ecosystem and its subcomponents, based on the theories presented by Chang and West. The outcome we achieve in this paper is concluded as below:

This paper proposes and explains the concept of Digital Ecosystem and its subclasses - Species and Environment. In addition, the subclasses of Species - Leader and Follower, and the subcomponents of Environment - six different categories of environments are explained through the use of the notation system introduced to substitute UML. Finally, we implement the prototype in Protégé-owl.

The limitations in our research are drawn as below:

This paper chooses only one Digital Ecosystem theory as its research goal, which supports limited knowledge to researchers.

The ontological representation towards subcomponents of Environment Concept is brief and surface, which needs more detailed and deeper research $n$ in the future.

Our conceptual model has not been validated in the real world.

Thus, against the above drawbacks in our research, in the future works we will extend our ontology design to deeper substructure and combine the theory of Soluta.net to our prototype to create a unified and widely recognized concept model. Finally, we will test the model in the real world by other researchers' comments.

\section{ACKNOWLEDGMENT}

We would like to express my gratitude to Professor Chang and Dr. West for their key note at iiWAS06 and we also appreciate the assistance of DEBII.

\section{REFERENCES}

[1] E. Chang, T. S. Dillon and F.K. Hussain, Trust and Reputation for Service Oriented Environments-Technologies for Building Business Intelligence and Consumer Confidence, Brisbane: John Wiley \& Sons, 2005, pp.131-153.

[2] E. Chang, M. Quaddus and R. Ramaseshan, "The vision of DEBI Institute: Digital Ecosystems and business intelligence," unpublished, 2006.

[3] P. Ferronato, "Introduction to the Digital Business Ecosystem project," presented at MDA Technical Forum, Tokyo, 2004.

[4] E. Chang and M. West, "Digital Ecosystem - A next generation of the collaborative environment," presented at iiWAS 2006, Yogyakarta, 2006.

[5] N. Guarino, "Ontology-Driven Conceptual Modeling," presented at 21st International Conference on Conceptual Modelling, Tampere, 2002, p.10. 